\title{
A level-1 pixel based track trigger for the CMS HL-LHC upgrade
}

\author{
Chang-Seong Moon ${ }^{* \dagger}$ \\ Universidade Estadual Paulista (UNESP) \\ E-mail: csmoondern.ch
}

We present feasibility studies to investigate the performance and interest of a Level-1 trigger based on pixels. The Level-1 (real-time) pixel based tracking trigger is a novel trigger system that is based on real-time track reconstruction algorithms able to cope with very high rates and high flux of data in a very harsh environment. The pixel detector has an especially crucial role in precisely identifying the primary vertex of rare physics events from the large pile-up of events. The goal of adding the pixel information already at the real-time level of the selection is to help reducing the total Level-1 trigger rate while keeping a high selection capability. This is quite an innovative and challenging objective for the upgrade of the experiments for the High Luminosity LHC.

38th International Conference on High Energy Physics

3-10 August 2016

Chicago, USA

\footnotetext{
* Speaker.

$\dagger$ (on behalf of the CMS Collaboration. This material is based upon work supported by the Sao Paulo Research Foundation (FAPESP) under Grant No. 2013/01907-0 and 2014/17361-9.
} 


\section{Introduction}

The online trigger system at CMS selects interesting events recorded by the detector for offline storage and analysis. The trigger is hierarchically divided into a hardware-based part, the Level1 (L1) system, and a software-based part, the High-Level Trigger system (HLT). The L1 reads out the detector at full LHC frequency $(40 \mathrm{MHz})$ but coarse granularity, reducing the event rate to $\mathscr{O}(100 \mathrm{kHz})$; its output feeds the HLT, which has access to the full detector detector information and reduces the event rate further to $\mathscr{O}(1 \mathrm{kHz})$. The current L1 trigger at CMS is based on the calorimetric and muon chamber subdetectors, with no information from the tracking system being used. In the High Luminosity LHC (HL-LHC), a very high instantaneous luminosity $\left(5 \times 10^{34} \mathrm{~cm}^{-2} \mathrm{~s}^{-1}\right)$ is expected, and the average number of pile-up (PU) will be up to 140. As such, not only the overall rate of physics processes is bound to increase but the identification and selection of interesting physics events amongst the tens of PU collision products is bound to become more difficult. If no enhancements to the trigger system were made, the increase of the trigger energy thresholds would be the only way to keep the L1 rate under control, jeopardising the overall physics potential of the experiment. The main goal of the CMS L1 trigger in Phase 2 upgrade (around 2024) for HL-LHC is to avoid that scenario, overcoming the challenging conditions and keeping the trigger thresholds as low as possible. The L1 Track Trigger for the CMS Phase 2 upgrade is a new L1 component that helps achieve that goal. It consists of a self-seeded trigger based on the L1 Outer Tracker that can provide $\mathrm{L} 1$ tracks with $p_{\mathrm{T}}>2 \mathrm{GeV}$ and $\mathrm{z}$ coordinate vertex resolution of $1 \mathrm{~mm}$ [1].

\section{Motivation of the L1 Pixel Trigger}

We performed feasibility studies to investigate the potential performance gain of an additional L1 component based on the CMS silicon pixel detector, with the aim of improving the L1 Track Trigger. Both in offline and HLT reconstruction, the pixel detector has a crucial role in the precise determination of the primary vertex of important physics events from the large number of PU interactions. It stands to reason that the usage of the pixel information already at the real-time selection level would lead to a reduction of the total L1 trigger rate while keeping a high selection efficiency for interesting physics events. The L1 pixel-based tracking trigger (or simply, L1 Pixel Trigger) employs real-time track reconstruction algorithms that are able to cope with the very high event rates and high data flux in the harsh environment that is the HL-LHC.

In these studies, we show that the usage of pixel information can provide very high precision of vertex resolution at $\mathrm{L} 1$. We also show that a $\mathrm{L} 1$ electron trigger with pixels can help improve fake electron rejection while keeping high efficiency even in the very forward region. Furthermore, we show that complete L1 tracking - combining L1 outer track with pixel hits - can allow to perform $b$ jet identification (b tagging) at L1, increasing the probability of triggering on rare physics processes with $b$ jets in final state. This improved selection capability with $\mathrm{L} 1$ pixel information can be useful both for low-energy rare physics processes, such as Higgs pair production and Higgs production associated with a top quark pair that will be used for precise measurements of the Higgs boson properties. Additionally, it may impact searches for new physics like new resonances decaying to $t \bar{t}$ and $H H$. 


\section{Simulation framework}

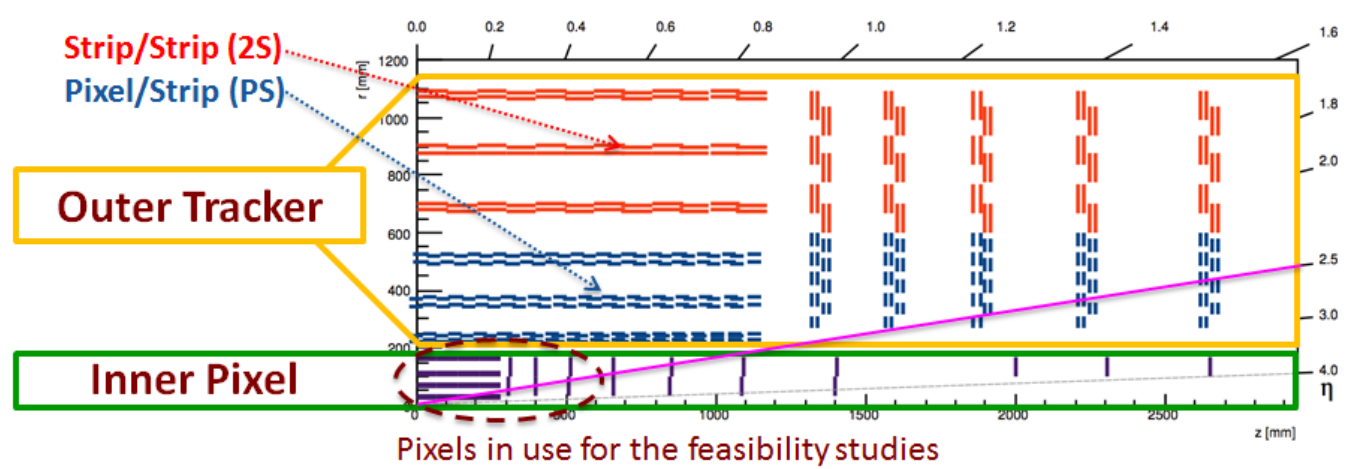

Figure 1: $r-z$ plane of Phase 2 tracking system with the L1 Outer Tracker as based on the flat barrel and endcap geometry design and the Pixel Detector

These feasibility studies are based on the current Phase 2 CMS simulation framework with L1 Outer Tracker and Pixel Detector as schematically shown in Fig. 1. We considered a pixel detector geometry with 4 barrel layers and 3 endcap disks, covering a range in $\eta$ up to 2.5; The calorimeter simulation is, in contrast with the tracker one, based on the Phase 1 proposal. Although this hybrid Phase 1-Phase 2 setup may underestimate the detector and trigger system capabilities, it provides an initial handle in their potential for Phase 2. A new and detailed simulation with final Phase 2 features is in preparation in CMS.

\section{Feasibility studies of the simulated L1 Pixel Trigger}

\subsection{L1 Pixel Electron Trigger}
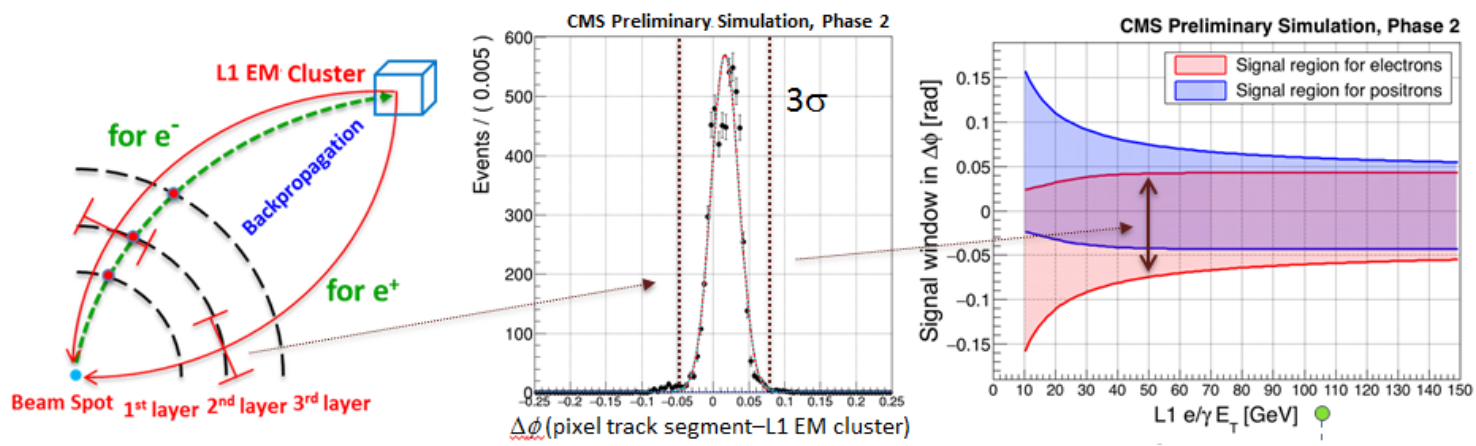

Figure 2: L1 Pixel Trigger strategy for electron

The pixel trigger strategy for electron identification is to get a seed from the L1 objects delivered by the electromagnetic calorimeter and match the pixel tracks with the L1 EM cluster in the region of interest [2] as described in Fig. 2. The choice of the matching variables and their window size lies at the core of the procedure of the pixel track algorithm matching with L1 EM cluster (PixTRK). The $\Delta \phi$ signal window of the PixTRK is designed separately for electrons (red 
curve) and positrons (blue curve). The signal window is obtained by matching between pixel track segment and L1 EM cluster and the $3 \sigma$ boundary of the Gaussian fit is determined as a function of L1 $e / \gamma$ transverse energy $\left(E_{\mathrm{T}}\right)$. The result was based on a single electron gun sample without PU.

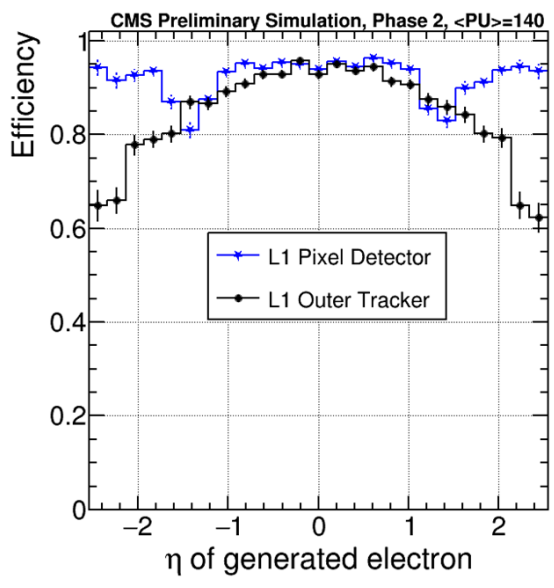

(a)

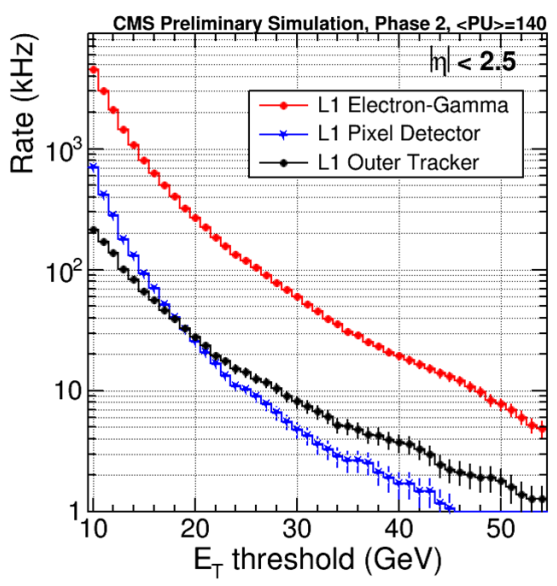

(b)

Figure 3: Electron trigger efficiency using the pixel detector (a): L1 single $e / \gamma$ trigger rate in minimum bias events with and without the pixel L1 trigger (b)

Figure 3a shows the efficiency of the L1 Pixel Electron Trigger measured as a function of the pseudorapidity $(\eta)$ of the generated electron. The efficiency is measured using a single electron sample with $\left\langle E_{\mathrm{T}}\right\rangle=35 \mathrm{GeV}$ and $\langle\mathrm{PU}\rangle=140$. For each event, the closest $(\Delta R<0.1) \mathrm{L} 1 e / \gamma$ object to the generated electron is selected. The efficiency is calculated for the $\mathrm{L} 1 e / \gamma$ objects: denominator is the number of selected $\mathrm{L} 1 e / \gamma$ objects with $\mathrm{L} 1 E_{\mathrm{T}}>20 \mathrm{GeV}$ and $|\eta|<2.5$, and numerator is the number of L1 $e / \gamma$ objects passing the PixTRK algorithm. The result is compared with the efficiency obtained by an algorithm matching L1 $e / \gamma$ objects to L1 tracks from the Outer Tracker [1], and applying an isolation requirement with respect to neighbouring L1 tracks. It can be seen that the L1 Pixel Trigger can keep a high efficiency of $90 \%$ in high $\eta$ regions, unlike the L1 Outer Tracker Trigger.

The L1 single $e / \gamma$ trigger rate as a function of $E_{\mathrm{T}}$ threshold is shown for $|\eta|<2.5$ in Fig. $3 \mathrm{~b}$. The rate is computed with a minimum bias sample with $\langle\mathrm{PU}\rangle=140$. The red curve shows the L1 single $e / \gamma$ trigger rate. The blue and black curves show the rates as obtained with the PixTRK algorithm and an algorithm matching to $\mathrm{L} 1$ tracks from the Outer Tracker. At $20 \mathrm{GeV} E_{\mathrm{T}}$ threshold, either L1 Pixel Detector or L1 Outer Tracker can reduce the L1 single $e / \gamma$ trigger rate by a factor of about 10 , corresponding to the $\mathrm{L} 1$ single $e / \gamma$ trigger $E_{\mathrm{T}}$ threshold lowered from $35 \mathrm{GeV}$ to $20 \mathrm{GeV}$ at $30 \mathrm{kHz}$. For the latter, an isolation requirement with respect to neighbouring L1 tracks is made. In addition, we estimated a factor of 2 better performance with single crystal level EM calorimeter.

\subsection{B tagging with the L1 Pixel Trigger}

The combined usage of the inner pixel and the outer tracker information allows a very precise estimate of the longitudinal component (i.e. along the beam axis) of the vertex, opening the possibility of performing $\mathrm{b}$ tagging at L1. We start with the $\mathrm{L} 1$ calorimeter jets for the first step of the $\mathrm{L} 1 \mathrm{~b}$ tagging. The $\mathrm{L} 1$ calorimeter jets are matched to $\mathrm{L} 1$ tracks from the outer tracker to determine 

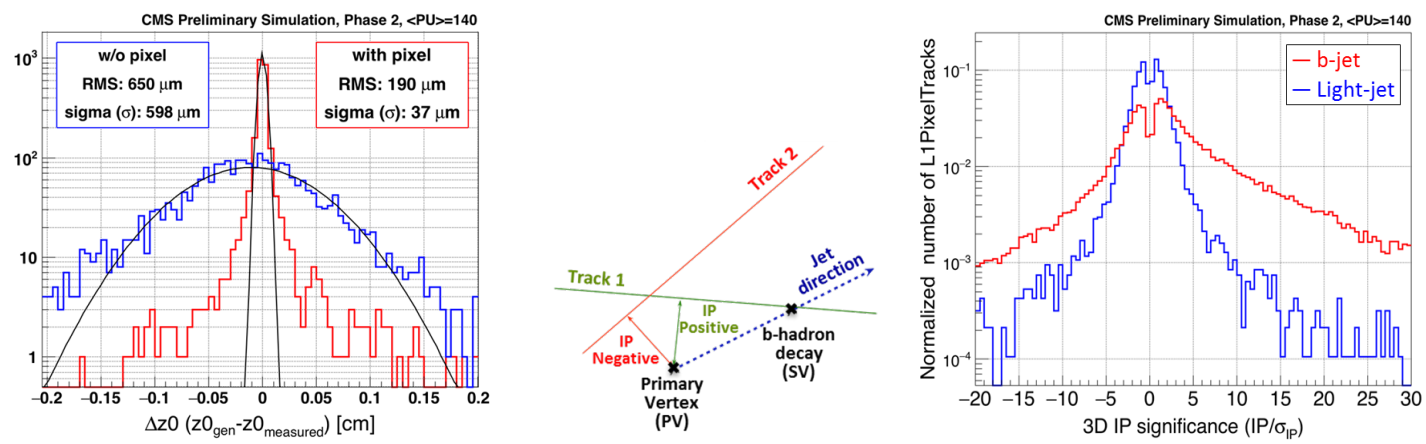

(a) Comparison of the jet vertex resolution (b) Schematic plot for the (c) 3D IP significance distributions for b jets between L1 tracks from the Outer Tracker 3D IP sign assignment (red) and light-flavour jets (blue) only (blue) and L1PixelTracks based on the Outer Tracker \& Inner Pixel (red).

Figure 4: L1 Pixel Track parameters

the $z$ position of the jet vertex [1]. The L1 tracks are filtered for $p_{\mathrm{T}}>2 \mathrm{GeV}$ in front-end, so $97 \%$ of the candidate tracks are low $p_{\mathrm{T}}$ tracks that are rejected. For these studies, L1 track-matched jets within $2 \mathrm{~mm}$ of the L1 primary vertex [1] are considered. L1 jets are also matched with generator level information to select only light-flavour jets. L1PixelTracks are formed by combining L1 tracks with matching pixel clusters and then repeating the linearized track fit. L1PixelTracks with $\Delta R<0.3$ and $\Delta z<2 \mathrm{~mm}$ from a L1 track-matched jet are selected. The L1PixelTracks are restricted to $p_{\mathrm{T}}>2 \mathrm{GeV}$ due to the $p_{\mathrm{T}}$ filtering in the front-end of the L1 Outer Tracker. An updated jet vertex is calculated from the $z_{0}$ position of the L1PixelTracks weighted by their $p_{\mathrm{T}}$.

The jet vertex resolution along the beam axis as provided by the L1 Outer Tracker is shown in the blue curve in Fig. 4a. The red curve shows the jet vertex resolution as determined using L1PixelTracks. The jet vertex resolution is defined by $\Delta z_{0}$ between the $z_{0}$ value of the hard interaction vertex at generator level and measured $z_{0}$ provided either by the L1 tracks or L1PixelTracks. The jet vertex resolution of $600 \mu \mathrm{m}$ as provided by the L1 Outer Tracker is improved to $40 \mu \mathrm{m}$ (Gaussian core) when including the pixels.

The significance of the three-dimensional impact parameter (3D IP) is used in this study as the main parameter to distinguish true $b$ flavour jets ( $b$ jets) from the light-flavour (udsg) jets. The 3D IP is obtained from the 2D IP in the transverse plane and the $z_{0}$ difference between the L1 jet and the L1PixelTrack as:

$$
3 D I P=\sqrt{(2 D I P)^{2}+\left(\Delta z_{0}\right)_{\text {L1 jet-L1PixelTrack }}^{2}}
$$

The 3D IP significance is then calculated as 3D IP divided by its uncertainty $\left(\mathrm{IP} / \sigma_{I P}\right)$ for each L1PixelTrack. The sign of the 3D IP is determined by the $\Delta \phi$ between the 2D IP and the jet direction in the transverse plane; if $\Delta \phi<\frac{\pi}{2}$, the sign of the 3D IP is assigned as positive, otherwise it is negative, as shown in Fig. $4 \mathrm{~b}$. The jet direction is computed from L1PixelTracks associated to the L1 jet. For this study, a sample of $\mathrm{b}$ jets and light-flavour jets is obtained from a sample of $t \bar{t}$ events, with $\langle\mathrm{PU}\rangle=140$ by matching the L1jets either with a Monte Carlo generator level $\mathrm{b}$ hadron or a light quark. A comparison of the 3D IP significance distributions between $b$ jets and light-flavour jets is shown in Fig. 4c. 


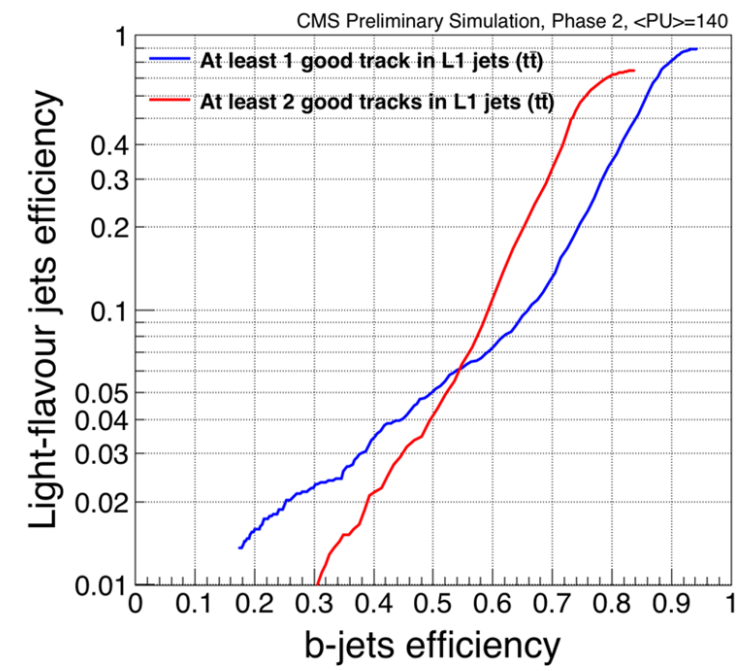

Figure 5: L1 $b$-tagging performance using at least one (blue) or two (red) good tracks

The $b$ tagging efficiency for $b$ jets versus that for light-flavour jets is computed using the track counting method based on requiring a minimum value of 3D IP significance. The $b$ jets / lightflavour jets efficiencies are defined as the number of L1 jets with at least 1 (blue curve) or 2 (red curve) L1PixelTrack(s) that satisfy 3D IP significance above a minimum threshold value, divided by the total number of L1 jets matched with generator level b hadron within $\Delta R<0.3$ for the b jets / not matched with $\mathrm{b}$ or $\mathrm{c}$ hadron using $\Delta R>0.3$ for light-flavour jets in $t \bar{t}$ events as shown in Fig. 5 . A light-flavour jet background efficiency of $10 \%$ is obtained for a $b$ jet signal efficiency of 60-65\% for $\mathrm{L} 1$ jet $p_{\mathrm{T}}>20 \mathrm{GeV}$ and $|\eta|<2.5$.

\section{Conclusion and outlook}

More feasibility and performance studies of the L1 Pixel Trigger are underway, including other studies for tau, muon and very forward physics capabilities. The preliminary results indicate potential benefits of the L1 Pixel Trigger, including a sizeable L1 trigger rate reduction while keeping high trigger efficiency and low $E_{\mathrm{T}}$ thresholds, for the electron case, and enhanced trigger capabilities for the $\mathrm{b}$ tagging case. These improvements may impact a large scope of the physics program at HL-LHC. It is clear that there are still technical challenges to be overcome, including those related to the L1 bandwidth and latency at the HL-LHC. Hardware-level developments are in progress on different fronts: the front-end ASIC studies in RD53, the design of the readout chain and integration in the L1 trigger architecture for the HL-LHC, and the development of the real-time algorithms and of platforms for testing and benchmarking.

\section{References}

[1] CMS Collaboration, Technical Proposal for the Phase 2 Upgrade of the CMS Detector, CERN-LHCC-2015-010.

[2] C.S. Moon and A. Savoy-Navarro, Level-1 pixel based tracking trigger algorithm for LHC upgrade, 2015 JINST 10 C10001. 